\title{
A Study on the Split Effect of Projectile Debris on Honeycomb Core
}

\author{
Zhiyuan Zhang, Runqiang Chi, Baojun Pang and Gongshun Guan \\ Hypervelocity Impact Research Center, Harbin Institute of Technology, Harbin, China
}

\begin{abstract}
Keywords: Space debris, Honeycomb sandwich panel, Split, Damage
Abstract. Split appeared after initial interaction between honeycomb core and projectile debris when projectile hypervelocity impact on honeycomb sandwich panel. In order to investigate the split characteristics of projectile debris, a 3D simulation model of projectile hypervelocity impact on honeycomb sandwich panel was established. The simulation model was validated with experimental test by comparing numerical experimental damage performance, it reveal better agreement with both simulation and experiment. The simulation results of split phenomenon and the damage of honeycomb core and rear facesheet show that split phenomenon disappears with time going, but it has a close relation to initial bifurcation pattern of projectile. The energy change trend of honeycomb core also demonstrated that damage was obviously influenced by location of projectile impact point.
\end{abstract}

\section{Introduction}

More than half a century, a series of achievements were got with the increase of human exploration of space activities, as well as a large amount of space debris were produced in earth orbit, which pose a serious threat to further space exploration [1]. In addition to being able to avoid large catalogued debris actively, tiny fragments of millimeter become the main research object of spacecraft protection. The speed of space debris impact the spacecraft is about $1 \sim 15 \mathrm{~km} / \mathrm{s}$, and a mass of complex phenomena accompanied in the process of the high speed impact [2-4]. Honeycomb sandwich panel has already become the "target" [5,6] of space debris strike because it is usually used as the outer wall of the spacecraft. Strike in honeycomb sandwich board panels produced a series of injuries, such as perforation, beat, tear, as well as honeycomb core wrinkle, fracture, disintegrate, etc. The process of space debris impact the front panel of honeycomb sandwich plate and formed debris cloud, and the process of debris cloud impacts honeycomb core and rear panel is unelasticity. The existing research is mainly focused on the damage of honeycomb core and panel [7, 8], and analyzing the form of projectile debris cloud from the side of the projectile motion path by using the flash X-ray radiography system [9] or numerical simulation[10, 11].

The position of hit point where spacecraft was struck is uncertainty due to the large number of space debris and the different distribution of the tracks. Some spacecraft use honeycomb sandwich as bulkheads. For this kind of spacecraft, honeycomb sandwich board panel formed different damage after the space debris impact.

Because of the complexity of the honeycomb sandwich panel structure and the limitations of high speed impact test, some special phenomena can't capture in the test. In order to solve this problem, the numerical simulation method provides an effective solution. Based on smoothed particle hydrodynamics (SPH) and the coupling of the finite element (FE) method, a numerical simulation model was established in this paper to simulate the process that projectile strike the honeycomb sandwich panel at a high speed, in order to research the morphology of debris cloud when point of impact is different.

\section{Numerical simulation model}

The numerical simulation model was established meanwhile implementing plentiful high-speed impact tests. The accuracy of this simulation model could be verified by these experimental results. Hypervelocity impact test

The experiments of projectile impact honeycomb sandwich panel were implemented by two-stage light-gas guns system in Hypervelocity Impact Research Center of Harbin Institute of Technology. The 
two-stage light-gas guns system mainly includes launch equipment, target cabin, laser velocity measurement system, flash X-ray radiography system, etc. It is hard to confirm the position of impact point in impact experiments. Through the comparison and analysis found that the approximate area of impact point is in the center of the honeycomb core, so that this experimental result was selected as a research object which compared with other experiments. The result which we selected was shown in Fig. 1. Table 1 shows the parameters of projectile and honeycomb sandwich panel, the whole size of honeycomb sandwich panel is $100 \mathrm{~mm} \times 100 \mathrm{~mm} \times 21.6 \mathrm{~mm}$. the velocity of projectile was $1.915 \mathrm{~km} / \mathrm{s}$. Fig. 1 shows the perforation diameter of rear facesheet is about $14 \mathrm{~mm}$, and the flanging height is about $5 \mathrm{~mm}$.

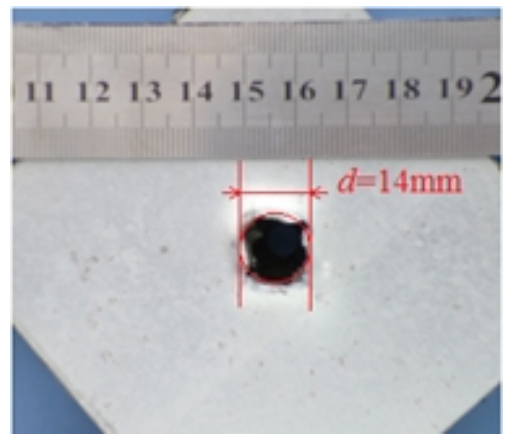

(a) The damage of rear facesheet

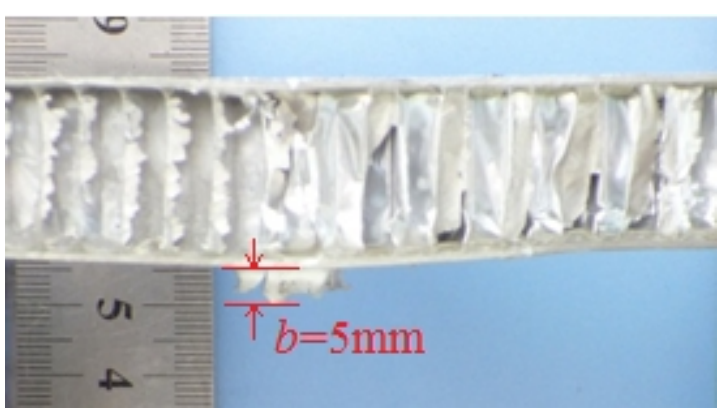

(b) Dimensional measurement

Fig. 1 The experimental result

In order to verify the accuracy of the material model and the geometric model more accurately, the model material and size are consistent with the honeycomb sandwich board material, thickness of the panel, honeycomb core thickness and height which were used in above experiment. Contrast test and numerical simulation results and then determine the accuracy of the model. Aluminum alloy projectile modeling process using the SPH method, honeycomb core sandwich panel modeling process using FE method. Literatures [12, 13] have already shown that SPH method can avoid the grid serious deformation problems signally which appear in FE method. Panel used entity unit, honeycomb core using shell element. The grid was refined to the size of $0.3 \mathrm{~mm}$ near the impact point area grid refinement, and outside area grid size is $0.8 \mathrm{~mm}$. The grid size of each honeycomb core and the panel were identical, the model as shown in Fig. 2. AUTODYN software was used to simulation of honeycomb sandwich panel high-speed impact. The simulation model is descripted as shown in Table 1 .

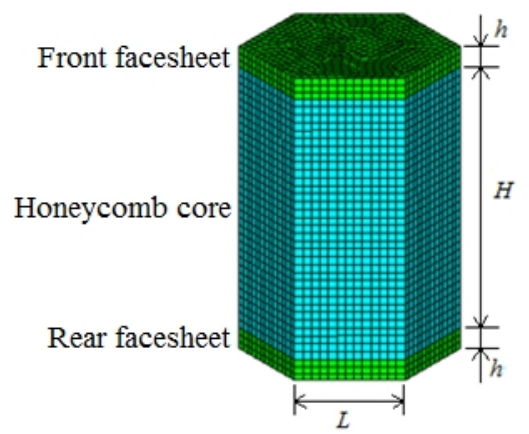

(a) Local grid

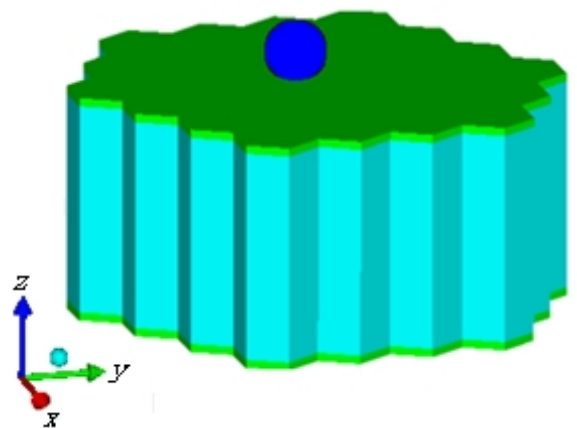

(b) Whole model

Fig. 2 Simulation model

Table1 Simulation target description

\begin{tabular}{|c|c|c|c|c|c|}
\hline & Material & Dimension/mm & EOS & Strength & Failure \\
\hline Projectile & Al 2017 & Sphere $/ D=5$ & Shock & Johnson Cook & $\begin{array}{c}\text { Principal } \\
\text { Stress }\end{array}$ \\
\hline $\begin{array}{l}\text { Facesheet } \\
\text { Honeycomb core }\end{array}$ & $\begin{array}{l}\text { Al 5A06 } \\
\text { Al 5A06 }\end{array}$ & $\begin{array}{c}\mathrm{h}=0.8 \\
\mathrm{~L}=4, \mathrm{H}=20, \mathrm{t}=0.025 \text { (Thickness }\end{array}$ & $\begin{array}{l}\text { Shock } \\
\text { Linear }\end{array}$ & $\begin{array}{l}\text { Johnson Cook } \\
\text { Johnson Cook }\end{array}$ & $\begin{array}{l}\text { Plastic strain } \\
\text { Plastic strain }\end{array}$ \\
\hline
\end{tabular}

The material non-linear was described by the Johnson Cook model. The yield stress $(\sigma)$ is expressed as follows:

$\sigma=\left(A+B \varepsilon^{n}\right)(1+C \ln \& \&)\left(1-T^{* m}\right)$ 
Where A、B、n、C and $m$ are the constants dependent on materials. $8 \&$ 为 is the plastic strain rate normalized with respect to a reference to a reference strain rate. $T^{*}=\left(T-T_{r}\right) /\left(T_{m}-T_{r}\right), T_{r}$ is the reference temperature and $T_{m}$ is the melting temperature. The properties used in the simulations are shown in Table 2.Plastic strain value is 0.39 in failure model.

Table 2 Material parameters used in the simulations

\begin{tabular}{cccccccc}
\hline & $A / \mathrm{MPa}$ & $B / \mathrm{MPa}$ & $n$ & $C$ & $m$ & $T_{m} / \mathrm{K}$ & Density $\mathrm{kg} \cdot \mathrm{m}^{-3}$ \\
\hline Al 5A06 & 235.4 & 622.3 & 0.58 & 0.0174 & 1.05 & 853 & 2.64 \\
$\mathrm{Al} 2017$ & 249.9 & 426.0 & 0.34 & 0.015 & 1.0 & 775 & 2.8 \\
\hline
\end{tabular}

The velocity of projectile in the numerical simulation model is $1.915 \mathrm{~km} / \mathrm{s}$ as well as experimental result. The impact point position is the honeycomb core center area which is consistent with the experimental result. The simulation results as shown in Fig. 3. The result illustrates that the projectile penetrated the honeycomb sandwich panel and the damage form is consistent with the experiment result, the flanging height is about $5 \mathrm{~mm}$ which compared with the experimental results are basically identical. The above result verified the accuracy of the geometric and material model established in this paper. So the simulation model established in this paper can be used in the research of damage characteristics which occurred in high-speed projectile impact honeycomb sandwich panel process.

In the following sections we will use this model to analyze the process of projectile impact honeycomb sandwich panel and the split effect of projectiles debris cloud.

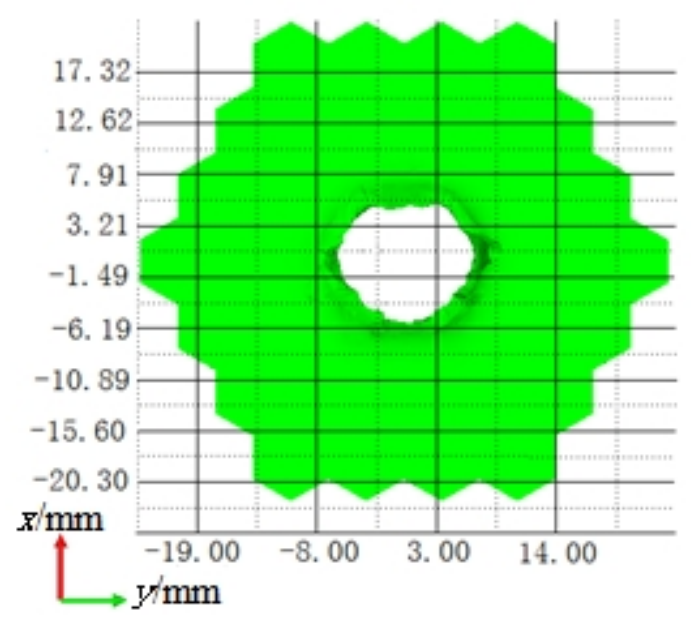

(a) The damage of rear facesheet

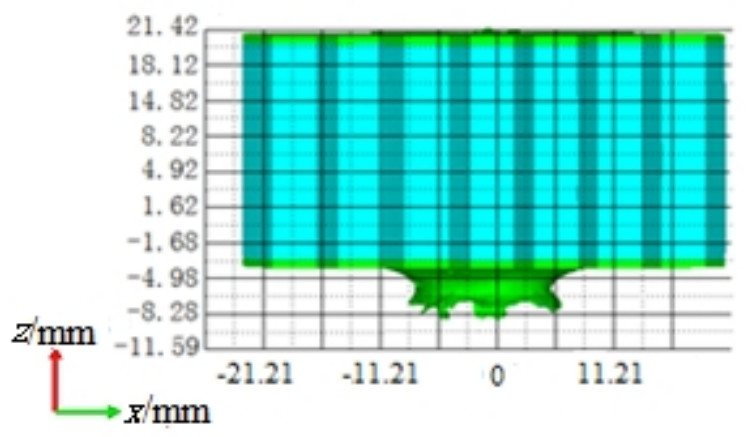

(b) Dimensional measurement

Fig. 3 Simulation result

\section{Validation of simulation model}

When the projectile impact honeycomb sandwich panel, due to the impact point position is different, meanwhile, the position of projectile debris cloud contact with honeycomb core is not the same. As shown in Fig. 4 is three impact points studied in this paper. $i$ is the center of the honeycomb core, $j$ is the middle point of public side of two adjacent honeycomb core, $\mathrm{k}$ is the common point of three adjacent honeycomb core. This three hit points $(i, j, k)$ represent the typical split effect point.

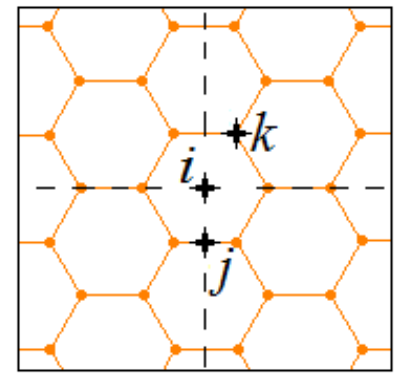

Fig. 4 Impact point of projectile 
When the projectile impact on point $i$, the projectile broken after through the front panel but keep the condition of forward movement along the incident direction of projectile in the form of debris cloud.

Because of the projectile debris cloud surrounded by cell wall, the radial diffusion of debris cloud was hindered and most of the debris cloud continues downward movement after gathered. Only part of the debris cloud radial diffused after through cellular wall. As shown in Fig. 5(a), Fig. 6(a) and Fig. 7(a) is debris cloud form in $6 \mu \mathrm{s}, 10 \mu \mathrm{s}$ and $15 \mu$ s respectively.

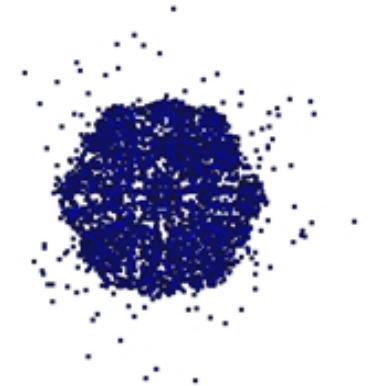

(a) $i$

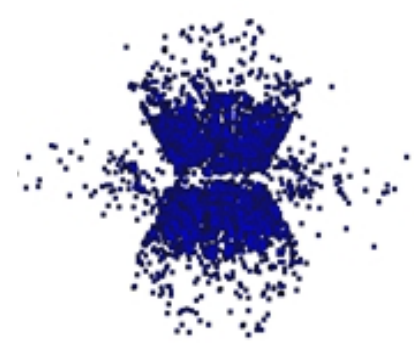

(c) $k$

(b) $j$

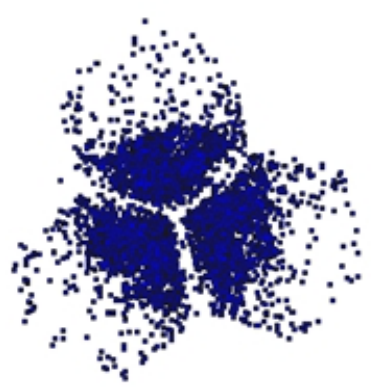

Fig. 5 Projectile debris of $6 \mu \mathrm{s}$

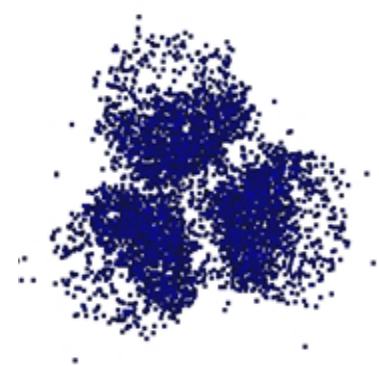

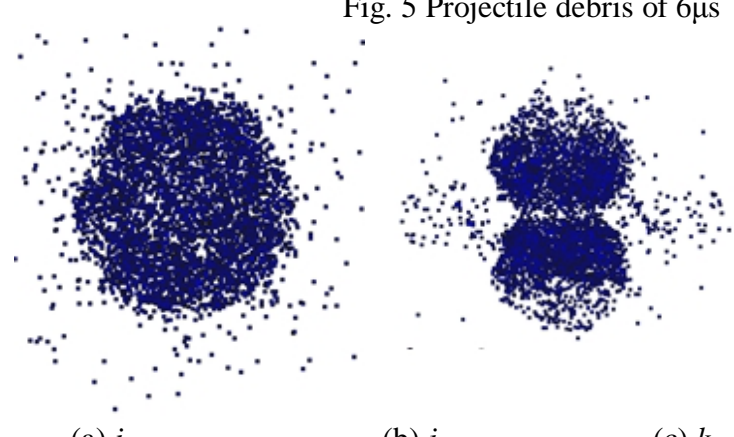

(a) $i$

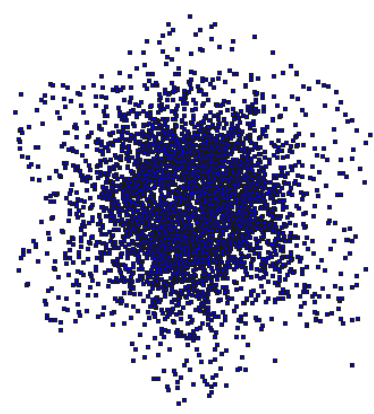

(a) $i$ (c) $k$

(b) $j$

Fig. 6 Projectile debris of $10 \mu \mathrm{s}$

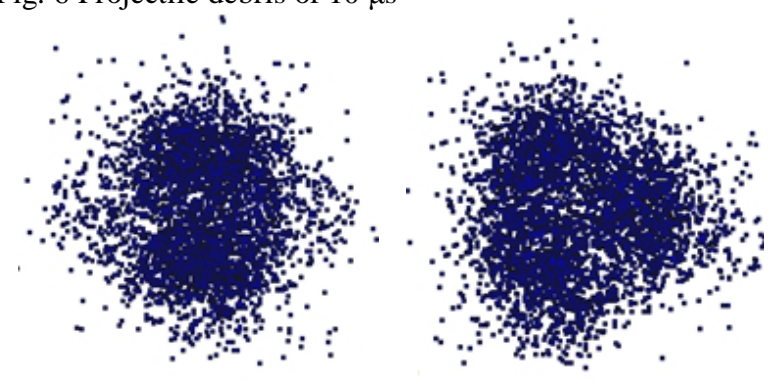

(b) $j$

Fig. 7 Projectile debris of $15 \mu \mathrm{s}$

When the impact point is $\mathrm{j}$, the center of the debris cloud hit the middle point of public side of two adjacent honeycomb core after the projectile penetrating the front panel, then the split phenomenon of projectile debris cloud occurred. The debris cloud forms in $6 \mu \mathrm{s}$, as shown in Fig. 5(b). Relative to the form of debris cloud in Fig. 5 (a), debris cloud appeared the split phenomenon in Fig. 5(b) as a result of the effect of cellular wall. This phenomenon shows that the projectile debris cloud shape has changed when the projectile debris cloud interact with cellular wall. Since there is only a cellular wall effect, the projectile debris cloud is divided into two.

When the impact point is $\mathrm{k}$, the debris cloud formed after the projectile penetrated the front panel, which interacted with cellular wall. At this moment, the impact position is the common point of three adjacent cellular wall, then the projectile debris cloud is divided into three splits due to the role of cell wall. Fig. 5 (c) shows the debris cloud form in $6 \mu$ s. As the projectile debris cloud continue to move, cellular wall have the effect to hinder the debris cloud continue to move. Debris cloud keeps downward movement and diffuses along the radial constantly at the same time, then debris cloud was rebound or move downward along the cellular wall after impact cellular wall if the cell wall has not been breakdown by debris cloud. 
It can be seen that split phenomenon of debris cloud becomes more and more fuzzy with the time pass by, finally disappear into a ball, by the Fig. 6 (b), (c) and 7(b), (c). This shows that short-term split phenomenon occurred in the process of projectile debris cloud interact with cellular wall: originally a ball of debris clouds, then occurred split phenomenon and finally disappear into a ball.

The different impact position not only leads to different forms of debris cloud, but also generates various damage shape of honeycomb core.

Due to the base area of a single honeycomb core is far greater than the planar projection area of the projectile, the projectile debris cloud did not directly contact with the cell wall after the projectile penetrating the front panel when the impact point is i. After a period of time, the projectile debris cloud began to contact the cell wall, then continue movement and diffusion of projectile debris cloud, the damage degree of the honeycomb core caused by the debris cloud, which is more and more serious, as shown in Fig. 8 (a).

When the impact point is $\mathrm{j}$, the projectile debris cloud contact and interact with the public cell surface of two adjacent cellular wall directly that lead to debris cloud split phenomenon appeared. With the diffusion process of the debris cloud, it is kinetic energy decreases gradually caused the damage to cellular wall decreases gradually, too. As shown in Fig. 8 (b).

When the impact point is $\mathrm{k}$, the projectile debris cloud contacts and interacts with three adjacent cellular wall, so the damage of honeycomb core is relatively serious which occurred a large area of rupture and collapse. Be similar to the impact point $\mathrm{j}$, the damage of cellular wall caused by projectile debris cloud decreased gradually with the continued movement of the projectile debris clouds, as is shown in Fig. 8 (c). It can be seen in Fig. 8 that projectile debris cloud was split by the cellular wall and then split of projectile debris cloud led to different damage forms of honeycomb core, as shown in Fig. 9 (a) is the damage form of back panel when impact point is i.

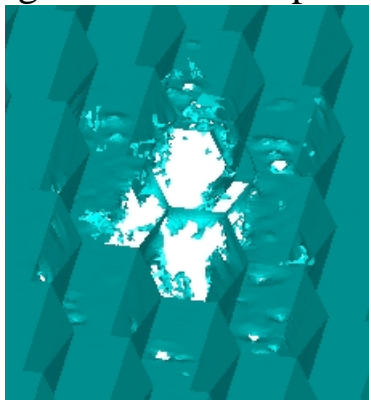

(a) $i$

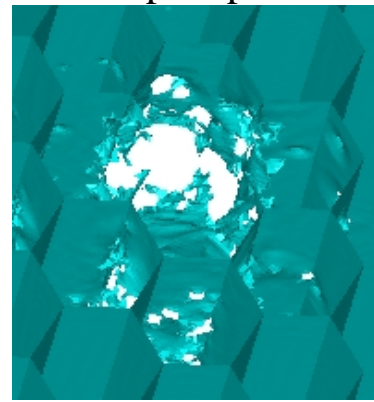

(b) $j$

(c) $k$

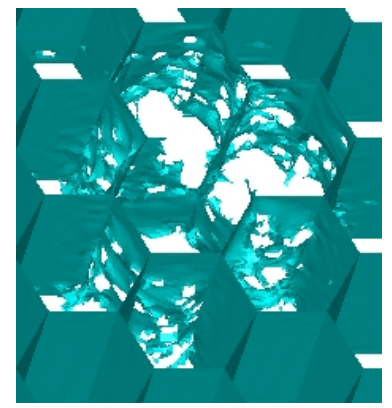

Fig. 8 Damage pattern of honeycomb core
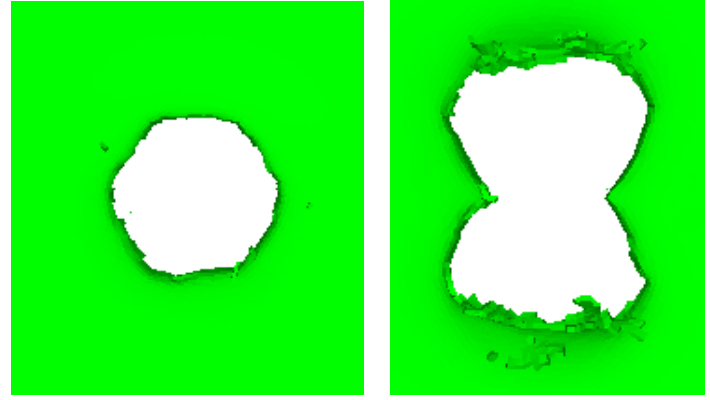

(b) $j$

(c) $k$

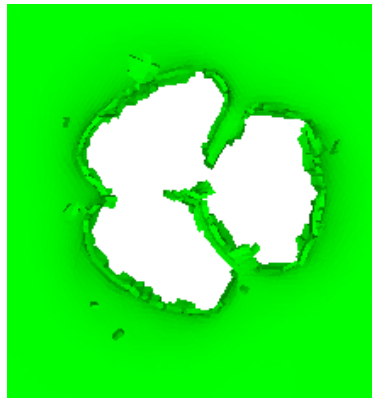

Fig. 9 Damage pattern of rear facesheet

The main part of the projectile debris cloud move within only a honeycomb core, so cellular wall played a role of gathering debris cloud and then forming a similar circular perforation in rear panel. Fig. 9 (b) is the damage form of rear panel when the impact point is j. Perforation shape like glyph "8" is caused by the split effect of projectile debris cloud.

Through Fig. 6 to Fig. 8 can see that although the projectile debris cloud had split phenomenon for a short time, the initial Split shape determines the impact characteristics of projectile debris cloud. 


\section{Conclusions}

The present paper aims to adopt the method of SHP and FE to research the phenomenon of a projectile impact on honeycomb sandwich plates with hypervelocity. Based on the simulation results, the following conclusions could be drawn.

1. The accuracy of numerical simulation model and material parameter selection were verified by comparison with high speed impact test results and the numerical simulation results.

2. The debris cloud formed by projectile penetrated the front panel of honeycomb sandwich panel occurred split phenomenon when debris cloud impacted honeycomb core and the split form is associated with the impact point position. When the impact point in the center of the honeycomb cores, does not appear split; when the impact point at the middle point of the public side of two adjacent honeycomb core, the debris cloud is divided into two petals; when the impact point at the public point of three adjacent honeycomb cores, the projectile debris cloud is divided into three Splits.

3. Split effect only to maintain a relatively short time which would disappear along with the extension of the impact time. According to the damage form of the honeycomb core and rear panel, you can see that this split effect doesn't disappear with the disappearance of the split phenomenon, and the initial debris cloud split form led to a different damage form of honeycomb core and rear panel.

\section{References}

[1] J.C.Liou and N. L.Johnson. Science, Vol. 311(2006), p. 340-341.

[2] G Drolshagen. Adv Space Res, Vol. 41(2008), p. 1123-1131.

[3] H.W. Li, J,W, Han, M.H. Cai and F.S. Wu. Acta Phys Sin, Vol. 62(2013), p. 1-8. In Chinese.

[4] H.W. Li, J,W, Han, J.G. Huang, M.H. Cai , X.Y. Li and Z. I. Gao. Acta Phys Sin, Vol. 59(2010), p. 1385-1390. In Chinese.

[5] E.A. Taylor, J.P. Glanville, R. A. Clegg and R.G. Turner. Int J Impact Eng, 2003, 29:691-702.

[6] J.M. Sibeaud, C. Thamié and C. Puillet. Int J Impact Eng, Vol. 35(2008), p. 1799-1807.

[7] M. Wicklein, S. Ryan and D.M. White. Int J Impact Eng, Vol. 35(2008), p. 1861-1869.

[8] S. Ryan, F. Schaefer and W. Riedel. Int J Impact Eng, Vol. 33 (2006), p. 703-712.

[9] M. Higashide, T. Koura and Y. Akahoshi. Int J Impact Eng, Vol. 35(2008), p. 1573-1577.

[10] P. Kang, S.K. Youn and J.H. Lim. Aerosp Sci Technol, Vol. 29(2013), p. 413-425.

[11] S. Ryan, F. Schaefer, M. Guyot, S. Hiermaier and M. Lambert. Int J Impact Eng, Vol. 35(2008), p. 1756-1763.

[12] M. Faraud, R. Destefanis, D. Palmieri and M.Marchetti. Int J Impact Eng, Vol.23(1999), p. 249-260.

[13] K.A. Alhussan, V.A. Babenko, I.M. Kozlov and A.S. Smetannikov. Int J Heat Mass Tran, Vol. 55(2012), p. 6340-6348. 